\title{
Prenatal antibiotic exposure and childhood asthma: a population-based study
}

\author{
Keely Loewen ${ }^{1,2}$, Barret Monchka ${ }^{3,4}$, Salaheddin M. Mahmud $3,4,5$, \\ Geert 't Jong ${ }^{1,6,7}$ and Meghan B. Azad (1) $1,5,6$
}

\begin{abstract}
Affiliations: 'Developmental Origins of Chronic Diseases in Children Network (DEVOTION), Children's Hospital Research Institute of Manitoba, Winnipeg, Canada. ${ }^{2}$ Max Rady College of Medicine, University of Manitoba, Winnipeg, Canada. ${ }^{3}$ Vaccine and Drug Evaluation Centre, Max Rady College of Medicine, University of Manitoba, Winnipeg, Canada. ${ }^{4}$ George and Fay Yee Centre for Healthcare Innovation, University of Manitoba, Winnipeg, Canada. ${ }^{5}$ Dept of Community Health Sciences, University of Manitoba, Winnipeg, Canada. ${ }^{6}$ Dept of Pediatrics and Child Health, University of Manitoba, Winnipeg, Canada. ${ }^{7}$ Dept of Pharmacology, University of Manitoba, Winipneg, Canada.
\end{abstract}

Correspondence: Meghan B. Azad, Children's Hospital Research Institute of Manitoba, 501G-715 McDermot Avenue, Winnipeg, Manitoba, Canada R3E 3P4. E-mail: meghan.azaddumanitoba.ca

@ERSpublications

Maternal antibiotic use is associated with childhood asthma, but the association is not specific to antibiotic use during pregnancy http://ow.ly/G5j230jAzs5

Cite this article as: Loewen K, Monchka B, Mahmud SM, et al. Prenatal antibiotic exposure and childhood asthma: a population-based study. Eur Respir J 2018; 52: 1702070 [https://doi.org/10.1183/13993003.020702017].

ABSTRACT Antibiotic use during infancy alters gut microbiota and immune development and is associated with an increased risk of childhood asthma. The impact of prenatal antibiotic exposure is unclear. We sought to characterise the association between prenatal antibiotic exposure and childhood asthma.

We performed a population-based cohort study using prescription records, hospitalisation records and physician billing claims from 213661 mother-child dyads born in Manitoba, Canada between 1996 and 2012. Associations were determined using Cox regression, adjusting for maternal asthma, postnatal antibiotics and other potential confounders. Sensitivity analyses evaluated maternal antibiotic use before and after pregnancy.

$36.8 \%$ of children were exposed prenatally to antibiotics and $10.1 \%$ developed asthma. Prenatal antibiotic exposure was associated with an increased risk of asthma (adjusted hazard ratio (aHR) 1.23, 95\% CI 1.20-1.27). There was an apparent dose response (aHR 1.15, 95\% CI 1.11-1.18 for one course; aHR 1.26, 95\% CI 1.21-1.32 for two courses; and aHR 1.51, 95\% CI 1.44-1.59 for three or more courses). Maternal antibiotic use during 9 months before pregnancy (aHR 1.27, 95\% CI 1.24-1.31) and 9 months postpartum (aHR 1.32, 95\% CI 1.28-1.36) were similarly associated with asthma.

Prenatal antibiotic exposure was associated with a dose-dependent increase in asthma risk. However, similar associations were observed for maternal antibiotic use before and after pregnancy, suggesting the association is either not directly causal, or not specific to pregnancy. 


\section{Introduction}

Asthma is the most common chronic disease of childhood [1], affecting $>10 \%$ of children worldwide [2]. In the United States, the total cost of asthma to society is estimated at USD 56 billion per year [3]. Similarly, in Canada asthma is a leading cause of healthcare utilisation, work absenteeism, lost productivity and diminished quality of life [4]. Given this large clinical and economic burden, and because there is no cure for asthma, it is important to identify modifiable risk factors to inform asthma prevention strategies.

The US Centers for Disease Control and Prevention report that antibiotics are prescribed at $12.6 \%$ of all ambulatory care visits, and $30 \%$ of these prescriptions may be unnecessary [5]. Across different settings, $20-40 \%$ of females are prescribed antibiotics during pregnancy [6-9], accounting for nearly $80 \%$ of all drugs used by pregnant females [6]. Prenatal antibiotic use may result in fetal exposure, as at least 11 types of broad-spectrum antibiotics cross the placenta, including penicillins, tetracyclines and lincosamides [10]. Mounting evidence shows that early-life exposure to antibiotics can have long-term health effects by perturbing the gut microbiota and disrupting immune system development [11-13]. However, relatively few of these studies have addressed antibiotic use during pregnancy, which could modify the maternal microbiota before its transmission to the fetus or infant during gestation, delivery, postnatal contact and lactation $[14,15]$.

Several longitudinal studies [16-18] and meta-analyses [19-21] indicate that antibiotic exposure during infancy is a risk factor for asthma (pooled OR 1.52, 95\% CI 1.30-1.77; 20 studies, $\mathrm{n}=685550$ ) [20], although some reports suggest confounding by indication or reverse causation [21,22]. Fewer studies have evaluated prenatal antibiotic exposure [23], with some [24-28] reporting increased asthma risk and others finding no association $[29,30]$. These studies have variably reported associations by antibiotic type [26, 27, 29], dose $[26,28]$ and trimester of exposure [27-29], but no single study has addressed all of these factors, and only a few have accounted for postnatal antibiotic use [25, 31]. A recent study by STокноLм et al. [30] found that maternal antibiotic use before, during and after pregnancy was similarly associated with asthma risk among offspring, suggesting that maternal propensity for infection (rather than antibiotic use per se) may be responsible for this association.

Using administrative health data capturing all children born in Manitoba, Canada from 1996 to 2012, we undertook a population-based study examining the association of maternal antibiotic use and childhood asthma. We classified antibiotics by type, number of courses and timing of exposure, and controlled for postnatal antibiotic use. In addition, we performed a sensitivity analysis to determine whether associations were specific to maternal antibiotic use during pregnancy.

\section{Patients and methods}

\section{Study design, population and data sources}

We conducted a retrospective cohort study of mother-infant dyads in Manitoba, Canada. Using Manitoba's health administrative data housed at the Manitoba Centre for Health Policy [32], we created a provincial birth cohort comprising all children born in Manitoba between 1996 and 2012. Data sources included physician billing claims, hospitalisation discharge abstracts and drug prescriptions collected by the Manitoba Health Services Insurance Plan (MHSIP) and the Drug Program Information Network (DPIN). The MHSIP and DPIN databases are reliable and valid data sources [33, 34]. Database record linkages were achieved through anonymised personal identifiers and a family registration number permitted linkage of maternal and child records. We included all dyads where linked maternal and child records were available, the mother was continuously registered with MHSIP for $\geqslant 1$ year before and $\geqslant 1$ year after pregnancy, and the child was continuously registered for $\geqslant 3$ years after birth $(\mathrm{n}=213661$ linked dyads from 235891 total eligible births; 90.6\%). Children without linked maternal data were less likely to develop asthma (incidence rate 8.74 versus 10.16 per 1000 person-years). This study was approved by the health research ethics board at the University of Manitoba and the Health Information Privacy Committee.

\section{Main exposure: maternal antibiotic use during pregnancy}

Maternal antibiotic use was determined from records of oral antibiotic prescriptions filled at community pharmacies and classified by dose (number of prescribed courses), timing (trimester of pregnancy, calculated from the infant's date of birth and accounting for gestational age) and type of antibiotic. All oral antibiotics were considered; they were grouped according to the Anatomical Therapeutic Chemical (ATC) classification system as follows: $\beta$-lactam penicillins (J01C); other $\beta$-lactams (J01D); macrolides, streptogramins and lincosamides (J01F); sulphonamides and trimethoprim (J01E); and tetracyclines (J01A), quinolones (J01M) and others (J01B, J01G, J01X). Antibiotics dispensed or administered in hospital are not captured in the DPIN database. 
Primary outcome: child asthma

Asthma was defined as meeting any of the following criteria after the age of 5 years: 1) any hospitalisation for asthma; 2) two or more physician diagnoses of asthma, $\geqslant 3$ months apart and within a 1-year period; or 3) two or more prescriptions for asthma medications within a 1-year period. The age requirement was applied because 5 years is the minimum age for confirming asthma diagnosis by lung function testing, and misdiagnosis is common before this age [35]. We used the index date (the date when the child first met any of the above criteria, which could be prior to age 5 years) to capture incident cases for survival analysis. This definition was based on the validated definition applied by KozYrSKYj et al. [17] using the same administrative database. We modified this definition to increase specificity by requiring repeated physician diagnoses to be $\geqslant 3$ months apart.

\section{Potential confounders}

The following potential confounders were documented from administrative health records: infant sex, residence location (urban or rural), length of gestation, number of siblings and maternal asthma (defined using the algorithm described earlier). Postnatal antibiotic exposure in the first year of life (any or none) was determined from infant prescription records.

\section{Statistical analysis}

We conducted a time-to-event analysis, measuring time to event from a child's birthdate to the earliest of the following dates: date the child first met the asthma diagnosis definition, death, loss to follow-up or the end of the study period (March 31, 2015). Associations between prenatal antibiotic exposure and

\begin{tabular}{|c|c|}
\hline Dyads $\mathbf{n}$ & 213661 \\
\hline Maternal age years & $27.6 \pm 5.9$ \\
\hline Infant birthweight g & $3493 \pm 619$ \\
\hline Gestational age weeks & $39.1 \pm 1.9$ \\
\hline \multicolumn{2}{|l|}{ Sex } \\
\hline Male & $109437(51.2)$ \\
\hline Female & $104224(48.8)$ \\
\hline \multicolumn{2}{|l|}{ Delivery method } \\
\hline Caesarean section & $41248(19.3)$ \\
\hline Vaginal & 172413 (80.7) \\
\hline \multicolumn{2}{|l|}{ Feeding method at birth } \\
\hline Fully breastfed & $108147(50.6)$ \\
\hline Partially breastfed & $60891(28.5)$ \\
\hline Formula-fed & $41026(19.2)$ \\
\hline Unknown & $3597(1.7)$ \\
\hline \multicolumn{2}{|l|}{ Residence location } \\
\hline Urban & $115604(54.1)$ \\
\hline Rural & 97549 (45.7) \\
\hline Unknown & $508(0.2)$ \\
\hline \multicolumn{2}{|c|}{ Number of children in household } \\
\hline 1 & 80939 (37.9) \\
\hline 2 & 71403 (33.4) \\
\hline 3 & $34234(16.0)$ \\
\hline$\geqslant 4$ & $26842(12.6)$ \\
\hline Unknown & $243(0.1)$ \\
\hline \multicolumn{2}{|l|}{ Maternal asthma } \\
\hline Yes & $12780(6.0)$ \\
\hline No & $200881(94.0)$ \\
\hline \multicolumn{2}{|l|}{ Income quintile } \\
\hline Q1 (lowest) & 56019 (26.2) \\
\hline Q2 & $44214(20.7)$ \\
\hline Q3 & $39797(18.6)$ \\
\hline Q4 & 39017 (18.3) \\
\hline Q5 (highest) & $34106(16.0)$ \\
\hline Unknown & $508(0.2)$ \\
\hline
\end{tabular}

Data are presented as mean \pm SD, or $n(\%)$, unless otherwise stated. 
childhood asthma were estimated using Cox regression models and reported as crude and adjusted hazard ratios (HR and aHR, respectively) and 95\% confidence intervals, with adjustment for known asthma risk factors (e.g. male sex, maternal asthma) and confounders that either changed the crude estimates appreciably or were considered confounders a priori (e.g. antibiotic use in infancy, socioeconomic status). We conducted sensitivity analyses examining maternal antibiotic use during the 9-month window before and after pregnancy (defined based on the infant's birth date and gestational age). We modelled interaction terms to test for effect modification by infant sex, method of birth and newborn feeding method, and tested for the significance of including interaction terms using likelihood ratio tests.

\section{Results}

Our study population consisted of 213661 mother-child dyads with a median follow-up time of 9.3 years from birth. The mean maternal age was $27.6 \pm 5.9$ years and $6.0 \%$ of mothers had asthma (table 1). The majority (54.1\%) lived in urban settings and $37.9 \%$ of children were first-born. Overall, $36.8 \%$ of mothers received antibiotics during pregnancy, $45.2 \%$ of infants received antibiotics in their first year of life, and $10.1 \%$ of children developed asthma (incidence rate 10.2 per 1000 person-years) (tables 2 and 3). Maternal antibiotic use varied slightly by trimester, from $16.2 \%$ in the first trimester to $18.4 \%$ in the second trimester and $14.7 \%$ in the third trimester. The majority of mothers receiving antibiotics were prescribed a single course $(22.1 \%$ of all mothers) while fewer received two $(8.4 \%)$ or more $(6.2 \%)$ courses during their pregnancy. $\beta$-lactam penicillins were the most commonly prescribed type of antibiotic $(24.6 \%)$, with fewer mothers receiving other $\beta$-lactams $(6.1 \%)$; macrolides, lincosamides or streptogramins $(7.1 \%)$; tetracyclines, aminoglycosides or quinolones (7.5\%); and sulphonamides or trimethoprim (2.6\%).

Mothers with asthma were more likely to use antibiotics during pregnancy $(56.2 \%$ versus $35.5 \%$ among mothers with versus without asthma, $\mathrm{p}<0.0001)$, and their children had a higher rate of asthma $(21.0$ versus 9.6 cases per 1000 person-years, $\mathrm{p}<0.0001$ ) (table 3). Preterm birth and infant antibiotic use were positively associated with increased maternal antibiotic use and child asthma. In contrast, rural residence location and higher birth order were both positively associated with maternal antibiotic use, but inversely associated with child asthma. These potential confounders were included in multivariable models to determine the independent association of maternal antibiotic use and child asthma (table 4).

Children born to mothers receiving antibiotics during pregnancy had significantly higher rates of asthma (11.9 per 1000 person-years, 95\% CI 11.6-12.1) compared to their unexposed counterparts (9.2 per 1000 person-years, 95\% CI 9.0-9.4) (table 3) (HR 1.29, 95\% CI 1.26-1.33). This association persisted

\section{TABLE 2 Maternal and infant antibiotic use in Manitoba, Canada, 1996-2012}

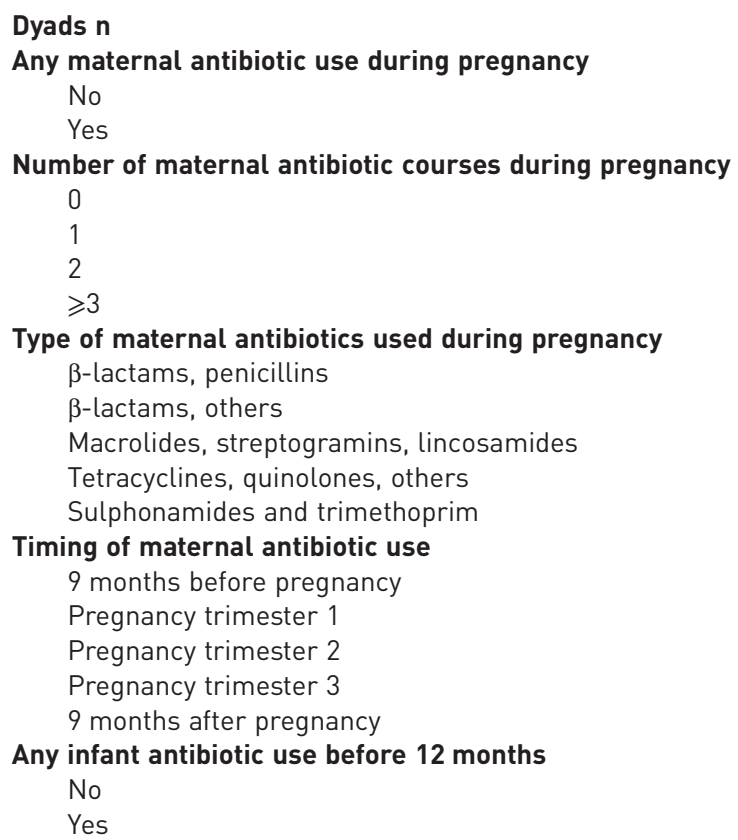

213661

$135139(63.2)$ 78522 (36.8)

$135139(63.2)$

$47286(22.1)$

$17954(8.4)$

$13282(6.2)$

$52598(24.6)$

$12998(6.1)$

$15120(7.1)$

$16074(7.5)$

$5615(2.6)$

$75166(35.2)$

$34562(16.2)$

$39340(18.4)$

31330 (14.7)

75761 (35.5)

$117136(54.8)$

96525 (45.2)

Data are presented as n (\%), unless otherwise stated. 
TABLE 3 Maternal antibiotic use and child asthma according to potential confounders in Manitoba, Canada, 1996-2012

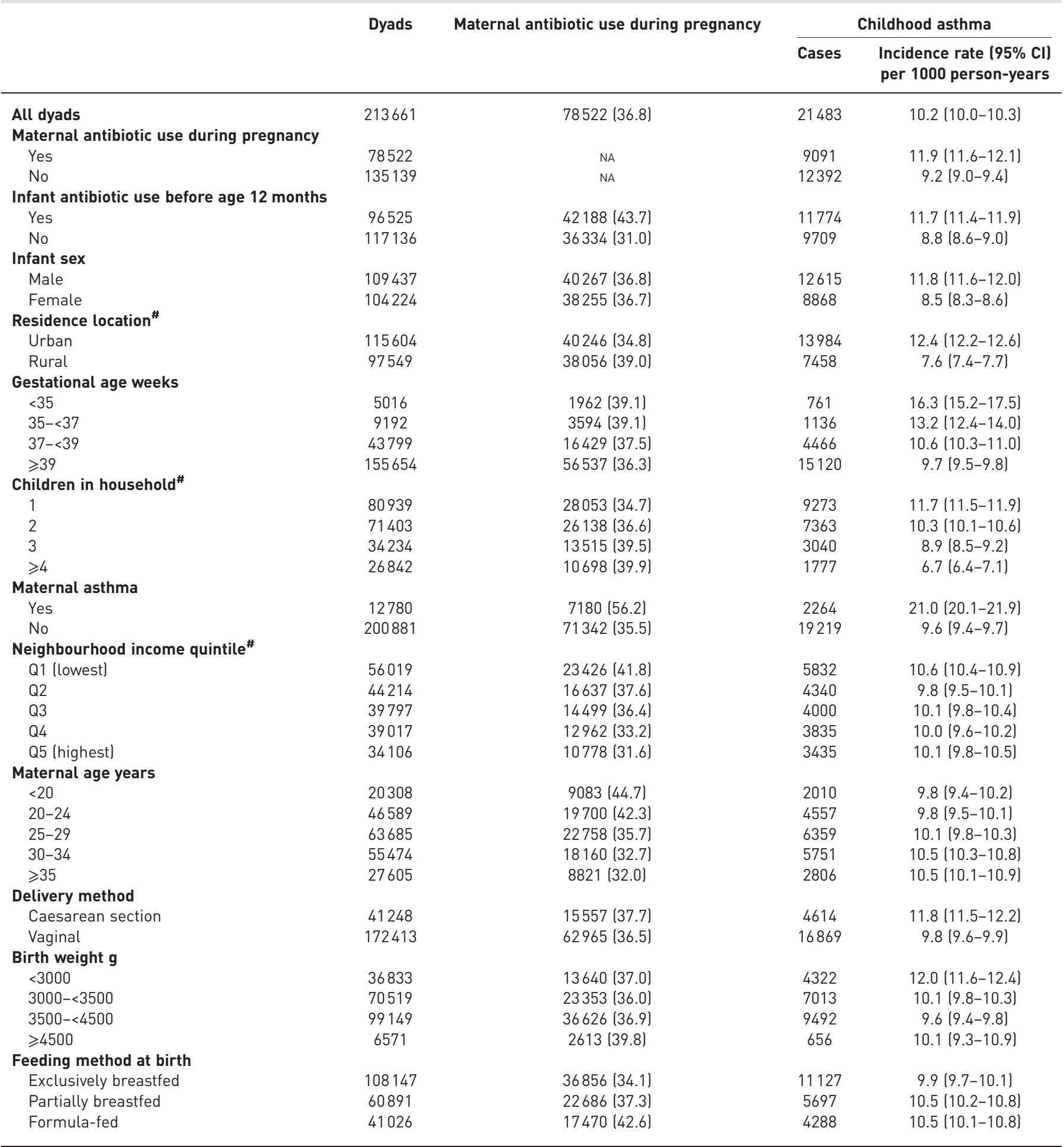

Data are presented as $n$ or $n(\%)$, unless otherwise stated. $n=213661$. NA: not applicable. ${ }^{\#}$ : excluding dyads with missing data, as reported in table 1.

following adjustment for sex, location of residence, gestational age, number of children in the household and maternal asthma (aHR 1.27, 95\% CI 1.24-1.31), and was unchanged by further adjustment for postnatal antibiotic exposure (aHR 1.23, 95\% CI 1.20-1.27) (table 4). There was no evidence of effect modification by infant sex, mode of delivery or infant feeding method (results not shown; $\mathrm{p}$ for interactions $>0.40$ ). 
An apparent dose response was observed, demonstrating progressively increasing asthma risk with each additional course of maternal antibiotics during pregnancy: aHR 1.15 (95\% CI 1.11-1.18) for one exposure, aHR 1.26 (95\% CI 1.21-1.33) for two exposures and aHR 1.51 (95\% CI 1.44-1.59) for three or more exposures (table 4, figure 1). When classified by type, most antibiotics were similarly associated with child asthma (table 4, figure 1), including $\beta$-lactam penicillins (aHR 1.22, 95\% CI 1.18-1.25); macrolides, lincosamides and streptogramins (aHR 1.21, 95\% CI 1.15-1.27); and sulphonamides and trimethoprim (aHR 1.28, 95\% CI 1.19-1.37). However, other $\beta$-lactams (aHR 0.99, 95\% CI 0.94-1.05) and tetracyclines, aminoglycosides and quinolones (aHR 1.06, 95\% CI 1.01-1.12) were not significantly associated with child asthma.

The timing of maternal exposure did not modify the association of maternal antibiotic use and child asthma. Associations were similar for maternal antibiotic use during the first trimester (aHR 1.18, 95\% CI 1.14-1.23), second trimester (aHR 1.15, 95\% CI 1.11-1.19) and third trimester of pregnancy (aHR 1.18, 95\% CI 1.13-1.22) (table 4). They were also similar for maternal antibiotic use during the 9 months before and after pregnancy (aHR 1.27, 95\% CI 1.24-1.31 and aHR 1.32, 95\% CI 1.28-1.36, respectively) (table 4, figure 1). Maternal antibiotic use before, during and after pregnancy was inter-correlated (online supplementary table S1); however sensitivity analyses excluding mothers who took antibiotics during more than one exposure period yielded similar results (table 5).

\section{Discussion}

In this population-based study, prenatal antibiotic exposure was associated with a $23 \%$ increased risk of asthma, independent of postnatal antibiotic exposure and several established asthma risk factors. There was an apparent dose response with repeated prenatal exposures; however, similar associations were observed for maternal antibiotic use before and after pregnancy. These results neither firmly support nor refute a directly causal pregnancy-specific relationship between maternal antibiotic use and childhood asthma; however, they contribute to the growing body of evidence linking early-life antibiotic exposure and asthma risk, and raise important questions for further research.

Our results are consistent with a case-control study by METSäLÄ et al. [26] showing that both prenatal and postnatal exposure to antibiotics were associated with an increased risk of asthma in Finnish children. In another case-control study, MULDer et al. [27] found that prenatal antibiotic exposure in the third

TABLE 4 Crude and adjusted estimates of the association between maternal antibiotic use and child asthma in Manitoba, Canada, 1996-2012

Crude HR for child asthma Adjusted HR for covariates ${ }^{\#}$

\begin{tabular}{ccc} 
& Adjusted HR for covariates ${ }^{\#}$ & $\begin{array}{c}\text { Adjusted HR for } \\
\text { covariates }{ }^{\#} \\
\text { infant antibiotics }\end{array}$ \\
\hline 213661 & 213418 & 213418 \\
1.00 (ref.) & 1.00 (ref.) & 1.00 (ref.) \\
$1.29(1.26-1.33)$ & $1.27(1.24-1.31)$ & $1.23(1.20-1.27)$ \\
1.00 (ref.) & 1.00 (ref.) & 1.00 (ref.) \\
$1.19(1.15-1.23)$ & $1.17(1.14-1.21)$ & $1.15(1.11-1.18)$ \\
$1.34(1.28-1.40)$ & $1.32(1.26-1.38)$ & $1.26(1.21-1.32)$ \\
$1.60(1.53-1.68)$ & $1.59(1.52-1.67)$ & $1.51(1.44-1.59)$ \\
& $1.25(1.22-1.29)$ & $1.22(1.18-1.25)$ \\
$1.25(1.21-1.28)$ & $1.01(0.95-1.07)$ & $0.99(0.94-1.05)$ \\
$0.95(0.89-1.00)$ & $1.24(1.18-1.30)$ & $1.21(1.15-1.27)$ \\
$1.35(1.29-1.42)$ & $1.07(1.02-1.13)$ & $1.06(1.01-1.12)$ \\
$1.11(1.06-1.17)$ & $1.31(1.22-1.40)$ & $1.28(1.19-1.37)$ \\
$1.30(1.21-1.39)$ & $1.31(1.28-1.35)$ & $1.27(1.24-1.31)$ \\
$1.39(1.35-1.43)$ & $1.21(1.17-1.25)$ & $1.18(1.14-1.23)$ \\
$1.26(1.22-1.30)$ & $1.18(1.14-1.22)$ & $1.15(1.11-1.19)$ \\
$1.20(1.16-1.24)$ & $1.20(1.16-1.25)$ & $1.18(1.13-1.22)$ \\
$1.14(1.10-1.19)$ & $1.37(1.34-1.41)$ & $1.32(1.28-1.36)$ \\
$1.42(1.38-1.46)$ & &
\end{tabular}

Data are presented as hazard ratio (HR) $(95 \% \mathrm{Cl})$, unless otherwise stated. $\mathrm{n}=213661$. " : covariates: maternal asthma, sex, location of residence, length of gestation, number of children in household and income quintile; ": any infant antibiotic use before 12 months of age. 


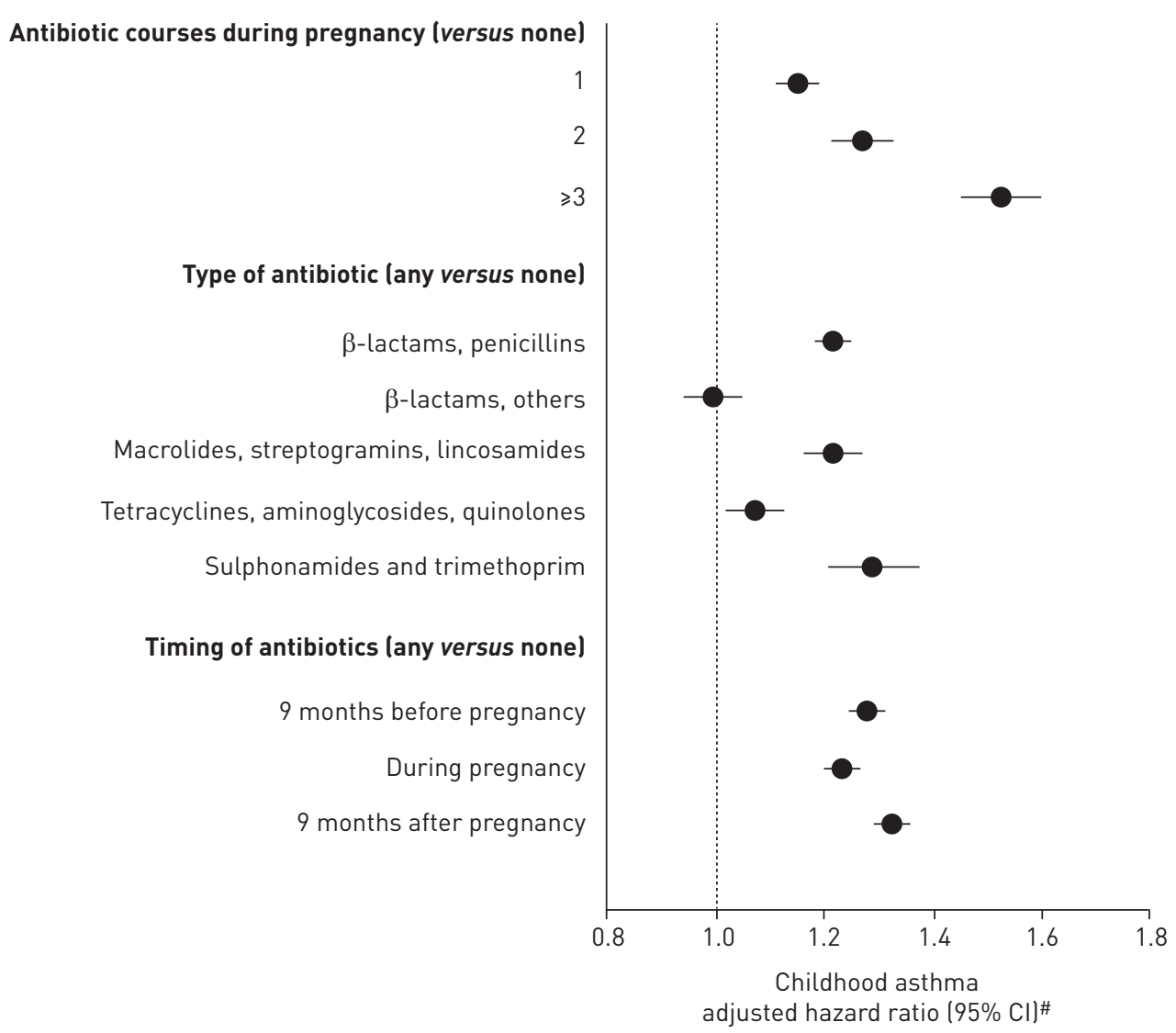

FIGURE 1 Associations between maternal antibiotics and childhood asthma by frequency, type and timing of antibiotic exposure among 213418 mother-infant dyads in Manitoba, Canada, 1996-2012. " : adjusted for maternal asthma, sex, location of residence, length of gestation, number of children in household and postnatal antibiotic exposure in the first year of life, as described in table 4.

trimester of pregnancy was associated with an increased risk of asthma in Dutch children, with consistent results in a case-sibling sensitivity analysis. While these studies seem to support a causal relationship between prenatal antibiotic exposure and asthma development, other studies have used different approaches and challenged this hypothesis. For example, ÖRTQVIST et al. [29] found that associations observed in Swedish children disappeared when using sibling controls, suggesting confounding by familial factors. In addition, Sтокноцм et al. [30] reported that maternal antibiotic use anytime from 80 weeks

TABLE 5 Sensitivity analysis for the association between maternal antibiotic use and child asthma in Manitoba, Canada, 19962012

\begin{tabular}{|c|c|c|c|c|}
\hline & \multicolumn{2}{|c|}{$\begin{array}{c}\text { Main analysis } \\
\text { Reference: no antibiotic use } \\
\text { during exposure period of interest }\end{array}$} & \multicolumn{2}{|c|}{$\begin{array}{c}\text { Sensitivity analysis }{ }^{\#} \\
\text { Reference: no antibitoic use } \\
\text { from } 9 \text { months before until } 9 \text { months } \\
\text { after pregnancy }\end{array}$} \\
\hline 9 months before pregnancy & 213418 & $1.31(1.28-1.35)$ & 115891 & $1.25(1.21-1.30)$ \\
\hline
\end{tabular}


prior to 80 weeks following pregnancy was similarly associated with childhood asthma in Denmark. The authors speculated that maternal propensity for infection (rather than antibiotic use) is the causal factor linking prenatal antibiotic exposure with asthma development. ÖRTQvist et al. [36] recently confirmed this phenomenon in Sweden, finding similar associations for maternal exposure before, during and after pregnancy. Consistent with Sтокноцм et al. [30] and ÖRTQvisT et al. [36], we have found that maternal antibiotic use before, during and after pregnancy is similarly associated with increased asthma risk in offspring. These results suggest the link between maternal antibiotic use and asthma in offspring is either not directly causal or not specific to pregnancy.

There are several potential explanations for a non-causal association between maternal antibiotic use and childhood asthma. First, the observed association may be confounded by healthcare utilisation patterns or other unmeasured factors that are shared within families, such as smoking and environmental exposures. Second, as Sтокноцм et al. [30] proposed, it is possible that maternal antibiotic use is a marker of genetic susceptibility to infections, which is inherited by offspring and imparts a predisposition for asthma. Third, as suggested by WeISS and LitonjuA [38], a maternal deficiency in vitamin D or other immunomodulatory nutrient could explain both the increased risk of infection in mothers and increased risk of asthma in offspring. Finally, since maternal and child medication usage are strongly associated [39], it is possible that maternal antibiotic use is a surrogate for infant antibiotic use, which is known to influence asthma development [17, 18]. While we could not address environmental exposures, genetics or nutritional hypotheses in our administrative database study, our findings do not support the final explanation, since our results were unchanged following adjustment for infant antibiotic use.

Another approach to address confounding in prenatal exposure studies is to evaluate fathers' exposures as a negative control. Mulder et al. [27] reported that maternal (but not paternal) antibiotic use during pregnancy was associated with child asthma at the age of 5 years, supporting a causal effect from in utero exposure. In contrast, ÖRTQVisT et al. [36] showed that both maternal and paternal antibiotic use during pregnancy were associated with child asthma before 2.5 years, suggesting confounding by shared familial factors. Notably, however, the maternal association was stronger and persistent throughout childhood, whereas paternal exposure was not associated with child asthma after 2.5 years. Thus, while we could not address paternal exposure in our study, this approach warrants further investigation.

Notably, the dose response observed by Sтокноцм et al. [30] and others [26, 28, 31], and replicated in our study, suggests that antibiotics or some related underlying factor (whether genetic, nutritional or environmental) may be causally related to asthma development in offspring. While this pattern could indicate dose response in a confounder, research is warranted to pursue these hypotheses in other settings where causal mechanisms can be explored and tested, such as clinical cohorts and animal models. It must also be acknowledged that, while the overuse of antibiotics can promote antimicrobial resistance and microbiome dysbiosis, untreated infections can also be harmful, especially to a developing fetus. For example, urinary tract infections during pregnancy are associated with intrauterine growth restriction, preterm labour and miscarriage [40,41]. Keeping this risk-benefit balance in mind, it is important to study and clarify the potentially unintended consequences of prenatal antibiotic exposure.

It is conceivable that maternal antibiotic use before, during and after pregnancy could impact infant microbiota and subsequent immune development. Pre-gestational antibiotic use may have long-term effects on the maternal microbiota that persist during pregnancy, and postpartum antibiotics could influence the transmission of maternal skin and breast milk microbiota to the infant [37]. Consistent with Mulder et al. [27], our finding that different types of antibiotics are differentially associated with child asthma lends support to this hypothesis, since different antibiotics will differentially impact maternal microbiota and their transmission to the infant. However, exposures occurring closer to the time of this microbial transfer would be expected to have a greater impact. The absence of any temporal gradient in our results, and those of Sтокноцм et al. [30], points to the involvement of additional mechanisms, as discussed later. Future research is needed to determine whether antibiotic-induced disruption of the maternal microbiota before pregnancy may persist during pregnancy or postpartum, whether postnatal maternal antibiotics impact maternal-infant sharing of microbes after pregnancy and whether these potential effects may influence asthma development.

Strengths of this study include the large unselected population, capturing virtually all children born in the province of Manitoba over an 18-year period, and the use of administrative healthcare data to objectively document asthma diagnoses, hospitalisations and antibiotic exposures. Using healthcare records eliminates recall bias, minimises loss to follow-up and provides key details that are not accurately captured by self-report, including the specific antibiotic type, dose and timing of exposure. Unlike most previous studies, we mutually adjusted our analyses for prenatal and postnatal antibiotic exposure during the first year of life; an important adjustment since maternal and infant healthcare utilisation tend to be correlated [39], but 
could be independently associated with child asthma development. In addition, we performed sensitivity analyses for maternal antibiotic use before and after pregnancy, although sibling controls and paternal exposures were not examined. Finally, our results confirm previous research [19] identifying maternal asthma, male sex, urban residence, premature birth, lower birth order, and antibiotic use during infancy as significant risk factors for asthma.

A limitation of our study is the lack of information about the indication for antibiotic treatment. In addition, exposure misclassification is possible since we cannot confirm patient compliance with filled prescriptions, and our database does not capture antibiotics administered in hospital. Thus, we could not account for intrapartum antibiotic prophylaxis for group B Streptococcus, which affects $>20 \%$ of deliveries in Manitoba [42] and has been shown to influence infant gut microbiota development [43]. In addition, we could not account for indirect exposure to antibiotics in food or the environment [44]. Outcome misclassification is also possible, since asthma is commonly misdiagnosed in young children; however, we evaluated multiple disease definitions and required evidence of serious (hospitalisation) or persistent (multiple diagnoses or prescriptions) disease after 5 years of age to maximise specificity. Finally, confounding bias is possible since we could not account for potential confounders that are not captured in administrative databases, such as maternal and child nutrition, education, smoking and environmental exposures including pets, tobacco smoke, mould and daycare attendance.

\section{Conclusions}

In this province-wide study, we observed a dose-dependent association between maternal antibiotic use and asthma risk in offspring; however, this association was not specific to antibiotic use during pregnancy. Further research is needed to better understand the nature of this association and address intrapartum antibiotic exposure. While our current results do not firmly support nor refute a directly causal pregnancy-specific relationship between maternal antibiotic use and childhood asthma, it remains important to use antibiotics judiciously.

Acknowledgements: The authors acknowledge the Manitoba Centre for Health Policy for the use of data contained in the Population Health Research Data Repository under project \#H2015:070 (HIPC\#2015/2016-03). The results and conclusions are those of the authors and no official endorsement by the Manitoba Centre for Health Policy, Manitoba Health or other data providers is intended or should be inferred. Data used in this study are from the Population Health Research Data Repository housed at the Manitoba Centre for Health Policy, University of Manitoba and were derived from data provided by Manitoba Health.

Conflict of interest: M.B. Azad reports grants (unrestricted research grants) from Heart and Stroke Foundation of Canada/Canadian Lung Association /Canadian Respiratory Research Network/Allergy, Genes and Environment Network of Centres of Excellence (co-funders), and from Children's Hospital Foundation of Manitoba, during the conduct of the study. S.M. Mahmud reports grants (unrestricted research grants) from GlaxoSmithKline, Sanofi Pasteur, Pfizer, Merck and Roche, outside the submitted work.

Support statement: This research was supported by the Children's Hospital Foundation of Manitoba, the Heart and Stroke Foundation of Canada, the Canadian Lung Association, the Canadian Respiratory Research Network, and the Allergy, Genes, and Environment (AllerGen) Network of Centres of Excellence. S.M. Mahmud holds a Canada Research Chair in Pharmacoepidemiology. M.B. Azad holds a Canada Research Chair in Developmental Origins of Chronic Disease. The analysis was conducted at the University of Manitoba Vaccine and Drug Evaluation Centre. Funding information for this article has been deposited with the Crossref Funder Registry.

\section{References}

$1 \quad$ Asher I, Pearce N. Global burden of asthma among children. Int I Tuberc Lung Dis 2014; 18: 1269-1278.

2 Pearce N, Ait-Khaled N, Beasley R, et al. Worldwide trends in the prevalence of asthma symptoms: phase III of the International Study of Asthma and Allergies in Childhood (ISAAC). Thorax 2007; 62: 758-766.

3 Bahadori K, Doyle-Waters MM, Marra C, et al. Economic burden of asthma: a systematic review. BMC Pulm Med 2009; 9: 24.

4 Ismaila AS, Sayani AP, Marin M, et al. Clinical, economic, and humanistic burden of asthma in Canada: a systematic review. BMC Pulm Med 2013; 13: 70.

5 Fleming-Dutra KE, Hersh AL, Shapiro DJ, et al. Prevalence of inappropriate antibiotic prescriptions among US ambulatory care visits, 2010-2011. JAMA 2016; 315: 1864-1873.

6 Bookstaver PB, Bland CM, Griffin B, et al. A review of antibiotic use in pregnancy. Pharmacotherapy 2015; 35: 1052-1062.

7 Broe A, Pottegård A, Lamont RF, et al. Increasing use of antibiotics in pregnancy during the period 2000-2010: prevalence, timing, category, and demographics. BJOG 2014; 121: 988-996.

8 de Jonge L, Jens Bos H, van Langen IM, et al. Antibiotics prescribed before, during and after pregnancy in the Netherlands: a drug utilization study. Pharmacoepidemiol Drug Saf 2014; 23: 60-68.

9 Santos F, Oraichi D, Bérard A. Prevalence and predictors of anti-infective use during pregnancy. Pharmacoepidemiol Drug Saf 2010; 19: 418-427.

10 Nahum G, Uhl K, Kennedy D. Antibiotic use in pregnancy and lactation: what is and is not known about teratogenic and toxic risks. Obstet Gynecol 2006; 107: 1120-1138. 
11 Arrieta M, Stiemsma LT, Dimitriu PA, et al. Early infancy microbial and metabolic alterations affect risk of childhood asthma. Sci Transl Med 2015; 7: 307ra152.

12 Kuo $\mathrm{CH}$, Kuo HF, Huang $\mathrm{CH}$, et al. Early life exposure to antibiotics and the risk of childhood allergic diseases: an update from the perspective of the hygiene hypothesis. J Microbiol Immunol Infect 2013; 46: 320-329.

13 Stokholm J, Schjørring S, Eskildsen CE, et al. Antibiotic use during pregnancy alters the commensal vaginal microbiota. Clin Microbiol Infect 2014; 20: 629-635.

14 Grönlund M, Gueimonde M, Laitinen $\mathrm{K}$, et al. Maternal breast-milk and intestinal bifidobacteria guide the compositional development of the Bifidobacterium microbiota in infants at risk of allergic disease. Pediatriya 2012; 52: 57-63.

15 Keski-Nisula L, Kyynäräinen HR, Kärkkäinen U, et al. Maternal intrapartum antibiotics and decreased vertical transmission of Lactobacillus to neonates during birth. Acta Paediatr 2013; 102: 480-485.

16 Hoskin-Parr L, Teyhan A, Blocker A, et al. Antibiotic exposure in the first two years of life and development of asthma and other allergic diseases by 7.5 yr: a dose-dependent relationship. Pediatr Allergy Immunol 2013; 24: $762-771$.

17 Kozyrskyj AL, Ernst PE, Becker AB. Increased risk of childhood asthma from antibiotic use in early life. Chest 2007; 131: 1753-1759.

18 Risnes KR, Belanger K, Murk W, et al. Antibiotic exposure by 6 months and asthma and allergy at 6 years: findings in a cohort of 1,401 US children. Am J Epidemiol 2011; 173: 310-318.

19 Castro-Rodriguez JA, Forno E, Rodriguez-Martinez CE, et al. Risk and protective factors for childhood asthma: what is the evidence? J Allergy Clin Immunol Pract 2016; 4: 1111-1122.

20 Murk W, Risnes KR, Bracken MB. Prenatal or early-life exposure to antibiotics and risk of childhood asthma: a systematic review. Pediatrics 2011; 127: 1125-1138.

21 Penders J, Kummeling I, Thijs C. Infant antibiotic use and wheeze and asthma risk: a systematic review and meta-analysis. Eur Respir J 2011; 38: 295-302.

22 Almqvist C, Wettermark B, Hedlin G, et al. Antibiotics and asthma medication in a large register-based cohort study - confounding, cause and effect. Clin Exp Allergy 2012; 42: 104-111.

23 Lamont HF, Blogg HJ, Lamont RF. Safety of antimicrobial treatment during pregnancy: a current review of resistance, immunomodulation and teratogenicity. Expert Opin Drug Saf 2014; 13: 1569-1581.

24 Collier CH, Risnes K, Norwitz ER, et al. Maternal infection in pregnancy and risk of asthma in offspring. Matern Child Health J 2013; 17: 1940-1950.

25 Lapin B, Piorkowski J, Ownby D, et al. Relationship between prenatal antibiotic use and asthma in at-risk children. Ann Allergy Asthma Immunol 2015; 114: 203-207.

26 Metsälä J, Lundqvist A, Virta LJ, et al. Prenatal and post-natal exposure to antibiotics and risk of asthma in childhood. Clin Exp Allergy 2015; 45: 137-145.

27 Mulder B, Pouwels KB, Schuiling-Veninga CCM, et al. Antibiotic use during pregnancy and asthma in preschool children: the influence of confounding. Clin Exp Allergy 2016; 46: 1214-1226.

28 Stensballe LG, Simonsen J, Jensen SM, et al. Use of antibiotics during pregnancy increases the risk of asthma in early childhood. J Pediatr 2013; 162: 832-838.

29 Örtqvist AK, Lundholm C, Kieler H, et al. Antibiotics in fetal and early life and subsequent childhood asthma: nationwide population based study with sibling analysis. BMJ 2014; 349: g6979.

30 Stokholm J, Sevelsted A, Bønnelykke K, et al. Maternal propensity for infections and risk of childhood asthma: a registry-based cohort study. Lancet Respir Med 2014; 2: 631-637.

31 McKeever TM, Lewis SA, Smith C, et al. The importance of prenatal exposures on the development of allergic disease: a birth cohort study using the West Midlands General Practice Database. Am J Respir Crit Care Med 2002; 166: 827-832.

32 Roos LL, Nicol PJ. A research registry: uses, development, and accuracy. J Clin Epidemiol 1999; 52: 39-47.

33 Kozyrskyj A, Mustard C. Validation of an electronic, population-based prescription database. Ann Pharmacother 1998; 32: 1152-1157.

34 Robinson J, Young T, Roos L, et al. Estimating the burden of disease: comparing administrative data and self-reports. Med Care 1997; 35: 932-947.

35 Global Initiative for Asthma (GINA). Global Strategy for Asthma Management and Prevention. Updated 2015 http:/ginasthma.org/wp-content/uploads/2016/01/GINA_Report_2015_Aug11-1.pdf

36 Örtqvist AK, Lundholm C, Fang F, et al. Parental antibiotics and childhood asthma - a population-based study. J Allergy Clin Immunol Pract 2017; 5: 1451-1454.

37 Blaser MJ, Bello MGD. Maternal antibiotic use and risk of asthma in offspring. Lancet Respir Med 2014; 2: e16.

38 Weiss ST, Litonjua AA. Maternal antibiotic use and childhood asthma: the missing link? Lancet Respir Med 2014; 2: $597-598$.

39 Janicke D, Finney J, Riley A. Children's health care use: a prospective investigation of factors related to care-seeking. Med Care 2001; 39: 990-1001.

40 Vazquez JC, Abalos E. Treatments for symptomatic urinary tract infections during pregnancy. Cochrane Database Syst Rev 2011; 1: CD002256

41 Harbison AF, Polly DM, Musselman ME. Antiinfective therapy for pregnant or lactating patients in the emergency department. Am J Health Syst Pharm 2015; 72: 189-197.

42 Persaud RR, Azad MB, Chari RS, et al. Perinatal antibiotic exposure of neonates in Canada and associated risk factors: a population- based study. J Matern Fetal Neonatal Med 2015; 28: 1190-1195.

43 Azad MB, Konya T, Persaud RR, et al. Impact of maternal intrapartum antibiotics, method of birth and breastfeeding on gut microbiota during the first year of life: a prospective cohort study. BJOG 2016; 123: 983-993.

44 Francino MP. Antibiotics and the human gut microbiome: dysbioses and accumulation of resistances. Front Microbiol 2016; 6: 1543. 